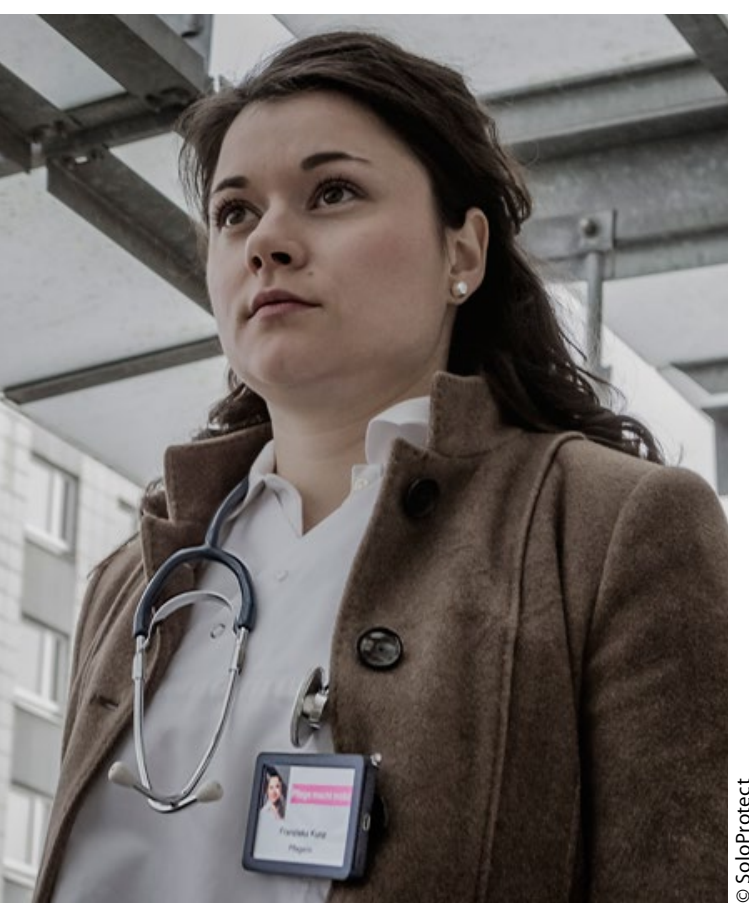

Ambulante Pflege

\section{Diskreter Schutz}

- Mitarbeiter von ambulanten Pflegediensten sind im Falle eines Unfalls, eines gesundheitlichen Notfalls oder eines tätlichen Übergriffs schutzlos und auf sich gestellt. Ein effektives und zuverlässiges Hilfsmittel für die Sicherheit von Alleinarbeitsplätzen ist die Personen-NotsignalAnlage "Identicom" des britischen Herstellers SoloProtect. Das angenehm zu tragende Gerät ist als leichtgewichtiger Fotoausweishalter gestaltet und ermöglicht dadurch ein von anderen Personen unbemerktes Auslösen eines Alarms. Das mit GPS ausgestattete Gerät arbeitet mit einer SIM-Karte und sucht sich automatisch das Mobilfunknetz mit dem besten Empfang. Dabei vereint das "Identicom" verschiedene Funktionen, die den Arbeitsalltag von Alleinarbeitern deutlich erleichtern. Mit dem Gerät ist rund um die Uhr ein zertifiziertes Alarmempfangszentrum verbunden, deren Mitarbeiter beim Auslösen des Alarms diskret über ein integriertes Mikrofon mithören, parallel alle Geschehnisse aufzeichnen, den Alleinarbeiter umgehend lokalisieren und für die angemessene Hilfeleistung sorgen. Der Notruf kann dabei sehr schnell mit nur einem Handgriff per Knopfdruck getätigt werden.

www.soloprotect.de
Chronische Obstipation

\section{Ein Prokinetikum auch für Männer}

— Seit kurzem können auch Männer mit chronischer Obstipation mit dem 5-HT4-Agonisten Prucaloprid behandelt werden, wenn Änderungen des Lebensstils und die Einnahme von Laxantien nicht helfen.

Per definitionem handelt es sich um Obstipation, wenn ein Patient seltener als jeden dritten Tag Stuhl entleeren kann. Doch Prof. Dr. Peter Layer, Hamburg, zufolge ist dies realitätsfremd: Eine Obstipation besteht dann, wenn sich der Patient obstipiert fühlt. Weniger die Stuhlfrequenz entscheidet darüber, ob die Lebensqualität leidet, sondern vielmehr die mit Obstipation verbundenen Begleitsymptome wie Völlegefühl, Blähungen oder die Notwendigkeit, die Entleerung manuell zu unterstützen. Als Krankheit werde Obstipation oft unterschätzt und den Patienten gern als "selbstverschuldet" angelastet. Da sie in verschiedenen Ländern der Welt ähnlich häufig auftritt, könne jedoch nicht von einer Zivilisationskrankheit gesprochen werden. Circa 14\% der Bevölkerung leiden unter chronischer Obstipation, wobei Frauen in den Statistiken etwa doppelt so häufig erfasst sind wie Männer. Dies, so Layer, gehe vermutlich darauf zurück, dass Männer die Störung oftmals bagatellisieren. Auch in den ursprünglichen Zulassungsstudien für Prucaloprid (Resolor ${ }^{\oplus}$ ) waren nur $12 \%$ der Teilnehmer männlich, was dazu geführt hat, dass die Europäische Arzneimittel-Agentur (EMA) die Zulassung zunächst nur für Frauen erteilte.

Gutes Nutzen-Risiko-Verhältnis: Prof. Dr. Ingolf Schiefke, Leipzig, stellte nun eine placebokontrollierte doppelt-verblindete Studie zur Wirksamkeit von Prucaloprid bei Männern vor, denen allgemeine Maßnahmen wie ausreichende Flüssigkeitszufuhr, Bewegung und ballaststoffreiche Ernährung sowie Laxantien nicht geholfen haben. Eingeschlossen waren 374 Männer, die über zwölf Wochen hinweg täglich 2mg Prucaloprid erhielten. Es zeigte sich ein ähnlich gutes Nutzen-Risiko-Verhältnis wie bei Frauen: Signifikant mehr Patienten der Verumgruppe als der Plazebogruppe ( 37,9 vs. $17,7 \% ; p<0,0001)$ hatten im Behandlungszeitraum mindestens drei spontane vollständige Stuhlentleerungen pro Woche und damit den primären Endpunkt erreicht. Besonders gut wirksam war Prucaloprid bei Slow-Transit-Formen.

(kvk)

Satellitensymposium "Chronische Obstipation - Welchen Einfluss hat das Geschlecht?"; Leipzig, 18.09.2015 (Veranstalter: Shire Deutschland GmbH)

Händehygiene

\section{Vorbilder gesucht}

— Um die Händehygiene-Compliance und damit den Infektionsschutz zu fördern, hat HARTMANN anlässlich des 50. Geburtstags des Hände-Desinfektionsmittels Sterillium eine besondere Aktion ins Leben gerufen. „Nennen Sie Ihren Modern Day Hero in der Hygiene" lautet die Initiative. Damit soll das Engagement von Mitarbeitern, die sich in Pflegeeinrichtungen und Krankenhäusern aktiv für eine bessere Händehygiene einsetzen, gewürdigt werden. Außergewöhnlich engagierte Mitarbeiter werden unter den Nominierungen ausgewählt und auf der Sterillium-Webseite vorgestellt. Dort berichten sie über ihre individuellen Herausforderungen bei der Händehygiene, persönliche Erfahrungen sowie besondere Ansätze zur Verbesserung der Compliance. Kennen Sie ein Vorbild auf dem Gebiet der Händehygiene an Ihrem Arbeitsplatz? Nominierungen unter: www.sterillium.de.

www.hartmann.de 